\title{
Methodology for the implementation of e-learning class during the COVID-19
}

\author{
Lina Montuori ${ }^{\mathrm{a}}$, Manuel Alcázar-Ortega ${ }^{\mathrm{b}}$, Carlos Vargas-Salgado $^{\mathrm{b}}$, Paula \\ Bastida-Molina ${ }^{c}$ \\ a Departamento de Termodinámica Aplicada, Universitat Politècnica de València, Camino de Vera, \\ s/n, edificio 5J, $2^{\mathrm{a}}$ planta. 46022 Valencia (España), lmontuori@upvnet.upv.es, ${ }^{\mathrm{b}}$ Departamento de \\ Ingeniería Eléctrica, Universitat Politècnica de València, Camino de Vera, s/n, edificio 5E, $2^{\mathrm{a}}$ planta. \\ 46022 Valencia (España), malcazar@iie.upv.es, carvarsa@upvnet.upv.es ${ }_{2}{ }^{\mathrm{c}}$ Instituto Universitario de \\ Investigación de Ingeniería Energética, Universitat Politècnica de València, Camino de Vera, s/n, \\ edificio 8E, acceso F, 2 $2^{\mathrm{a}}$ planta. 46022 Valencia (España), paubasmo@etsid.upv.es
}

\begin{abstract}
The serious situation caused by the coronavirus has forced Authorities to take drastic decisions that have affected the normal functioning of the entire society. One of the most impactful measures taken has been the self-discipline of the social distancing as the entire society has been obliged to stay at home.
\end{abstract}

At the education level, restrictions ordered by the Authorities have limited the access to all professors and students at the academic centers. In Spain, the state of alarm decreed by the Government has affected the entire Academic course and therefore, in order to be able to preserve the public service, the Polytechnic University of Valencia, in just a week, has been asked to revise the entire programs and settle on-line courses for more than 35.000 students in multiples disciplines. Within this framework, a methodology has been developed for the implementation of on-line learning courses in a period of crisis within a short time.

On-line learning has been demonstrated to be effective as face-to-face education, becoming one of the most popular higher education alternatives. However, students engaged in on-line classes result to be less engaged in collaborative learning, student-faculty interactions, and discussions with their classmates if compared to the traditional system. In this context, the barriers of on-line teaching classes have been investigated and tools to overcome them have been proposed. Finally, a real application to the Polytechnic University of Valencia is presented.

Keywords: Pandemic crisis, methodology, e-learning, university, education, COVID-19. 


\section{Introduction}

Due to the Covid-19, different measures have been adopted worldwide, some of which have affected in deep our habits and usual behavior. Social has been one of the core dimensions affected by the pandemic crisis, with measures to address it including social distancing (Fell, et al., 2020). It has implied that academic activities have had to move, in a very short time, from face-to-face classes to remote technics so as to make students not to lose the full academic year (Gewin, 2020). However, students ${ }^{\text {ee }}$ isolation may have a psychological impact on them, reducing their academic performance (Soland, et al., 2020).

In Spain, the Government declared the Alarm State on March 18 due to the coronavirus spread-out (Boletín Oficial del Estado, 2020), imposing the sudden closure of all educational facilities. In just one week, educators were asked to turn the face-to-face classes into on-line classes, so educational institutions reacted in different ways according to their technological development and level integration of IT tools. The analysis of external factors highlights that the COVID-19 strongly impacted on the usual organization of UPV (students and professors), not only for the mandatory switching from face-to-face to virtual work, but mainly due to the social distancing. The self-discipline obliged the entire society to stay at home, increasing the feeling of isolation and loneliness. Furthermore, the block of the commercial and industrial activities caused a social and economic crisis with the rising of the unemployment, reduction of employees ${ }^{\text {ee }}$ wages and the spread-out of people uncertainty and fear (Nicola, et al., 2020).

In this framework, a methodology has been developed for the implementation of online learning courses in a period of crisis within a short time. The methodology takes advantage from the ability of the $\mathrm{Z}$ generation students to deal with the new technologies, promoting the virtual collaboration "inter-pares". Thus, the limits of online teaching classes have been investigated and tools to overcome them have been proposed. Finally, a real application to the Polytechnic University of Valencia (UPV) is presented.

\section{E-Learning: Analysis and Methodology}

\subsection{Advanced e-learning methodology}

An innovative methodology for the implementation of the e-learning class upon the severe conditions caused by the pandemic crisis has been implemented. The effectiveness of the 
proposed methodology lies in the possibility to implement it in a very short time, using available information technology (IT) tools which are already in use in the educational institutions. The developed methodology is summarized in the figure 1 . The first phase is the strategic analysis of external factors that can impact on educational institutions and students learning. This phase allows to discover, evaluate and track macro-social, economic, technological and political factors and provides an overview of their crucial external influences on the educational organization and teaching methods.

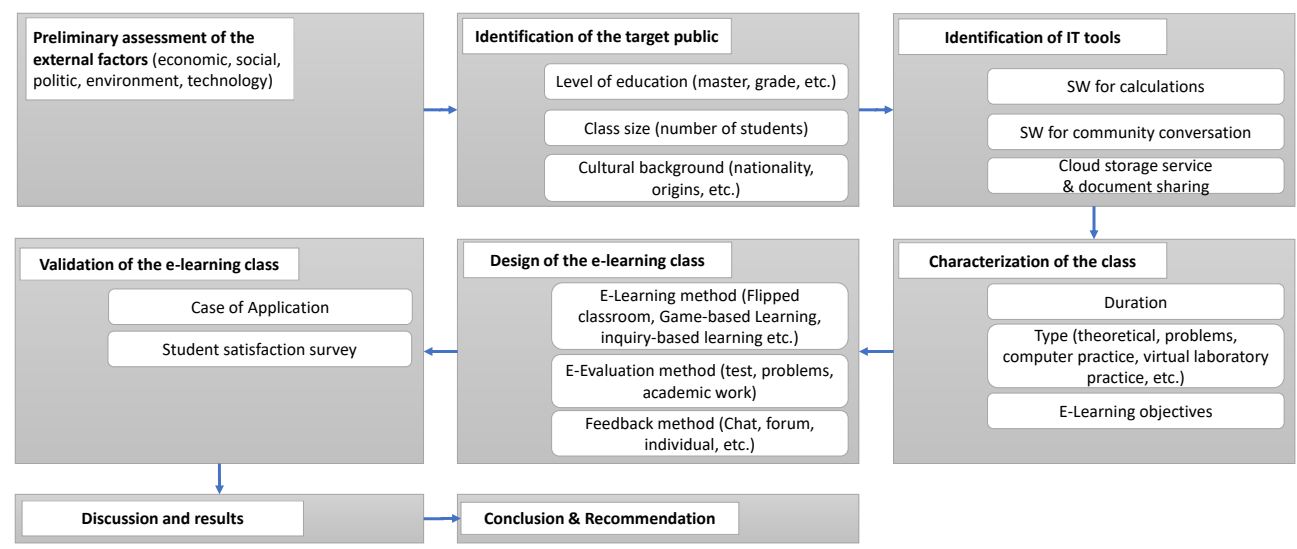

Fig. 1 Methodology for the implementation of e-learning class

Font: Authors

The second phase deals with the identification of the target public. The students ${ }^{\text {ee }}$ profile is determined according to their level of education (undergraduate, graduate, master) together with their cultural background (local or foreign student, etc.) and the class size (large, medium, small). The next phase deals with the identification of the available IT tools, already in use at the educational center that may be adopted to promote and support virtual classrooms.

After that, the structure of the class needs to be defined in terms of duration (hours per week and hours per day), type (theoretical classes, problems, virtual labs, etc.) and learning objectives that students should achieve. The following phase is focused on the design of the e-learning class with the definition of e-teaching, evaluation and feedback method, as well as the selection of the corresponding IT tools. Finally, the validation of the implemented e-learning class needs to be carried out with a real case of application. To do this, students ${ }^{\text {ee }}$ satisfaction is evaluated by means of a survey related to e-learning methods according their experience at UPV during the last semester. Finally, discussion and results are presented and some recommendation are suggested. 


\section{Case of Application}

The advanced e-learning methodology proposed here has been applied to the Master in Industrial Engineering at the Polytechnic University of Valencia (UPV) during the second semester of the a academic year 2019-2020 to face the pandemic crisis COVID-19. In this framework, the methodology has been applied to a group of 200 students. The average number of students per class is about 40 people. The UPV has deployed a high level information technology with a wide portfolio of IT tools for the implementation of on-line classes. For this application, virtual classes have been made through the community conversation tool „Microsoft Teams". For calculation and problem solving, different software have been applied, such as „Microsoft Excel"e, „Frío Atecyr" and „PVsyst". For the storage and document sharing, the ERP system currently in use in the UPV, „Poliformat", provides different efficient tools for sharing educational documentation and technical worksheets. The on-line classes had a mean duration of two hours with a frequency of two classes per week. Each class was split in one hour of theoretical concepts and one hour of problem solving. The e-learning objectives were the same as for the traditional classes, as presented in the Syllabus. Taking into account the previous steps, the design of an elearning class has been carried out. Virtual classes took place from the 23 March 2020 to the end of the Academic course in June. Classes were also recorded and uploaded to the cloud in order to allow students to re-watch them later. The educational documentation was prepared with the support of Microsoft Power Point, including slides and videos (screencasts). The daily learning level achieved by the student was tested by a game-based learning method through the collaborative app „Kahoot". This web application allows students to test their knowledge through a virtual competition with their classmates. To support the explanation during the class, the educator used an electronic blackboard that could be shared with students to actively collaborate and interact with them. During the all class, a chat box was available to students so as to share their doubts and to make questions. Feedback was constantly provided to students through the Poliformat ERP system. A dedicated "Forum" for the students was enabled in order to improve the communication and reduce the social isolation. The evaluation was carried out through Poliformat by using multiple-choice tests and problems be solved by computer and later uploaded throughout Poliformat. A scheme of the designed e-learning class is shown in the Figure 2.

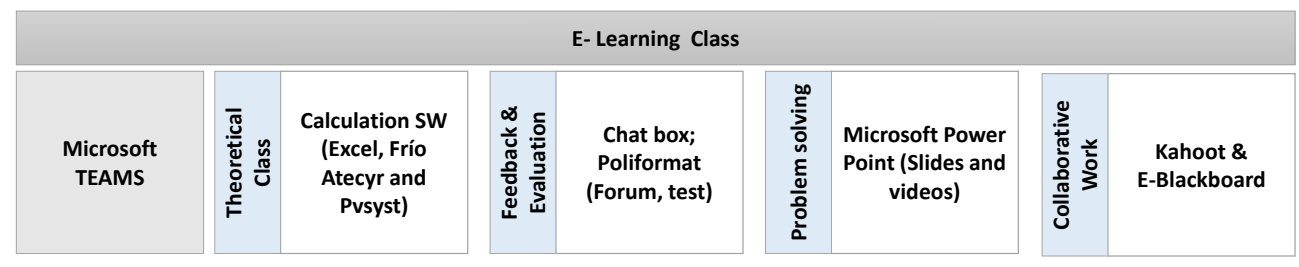

Fig. 2 Design of an e-learning class

Font: Authors 


\subsection{Student satisfaction survey}

At the end of e-learning course, students were asked to fill in an on-line survey of 10 questions. The questionnaire, specifically designed for this research, is shown in Table 1. The survey was sent to 200 students, replying 107 people as shown in Table 1. The satisfaction survey was aimed at understanding the feeling of the student during their participation to virtual classes. Furthermore, the importance given of the interaction between the professor and the students was investigated. In addition, the effectiveness of the implemented e-learning methodology was evaluated. Finally, pros and cons of the online class, as perceived by the students, have been identified and students ${ }^{\text {ec }}$ recommendations for improving the e-learning class have been collected.

Table 1. Student satisfaction survey

\begin{tabular}{llc}
\hline Questions & Answers & \% Replied \\
\hline Staying at home, does it facilitate the learning? & Dichotomist & $98 \%$ \\
\hline Importance of interacting with your classmates & Likert scale & $100 \%$ \\
\hline Importance of the teacher's presence for your learning & Likert scale & $100 \%$ \\
\hline Feeling of isolation during the on-line class & Likert scale & $100 \%$ \\
\hline Usefulness of recording on-line classes & Likert scale & $100 \%$ \\
\hline $\begin{array}{l}\text { Usefulness of collaborative and team work during on- } \\
\text { line classes }\end{array}$ & Likert scale & $98 \%$ \\
\hline $\begin{array}{l}\text { Recommended tools to promote student motivation } \\
\text { during the on-line class }\end{array}$ & $\begin{array}{l}\text { Multiple } \\
\text { choices }\end{array}$ & $98 \%$ \\
\hline Satisfaction level of participating in the on-line class & Open answer & $99 \%$ \\
\hline What did you like most about on-line classes? & Open answer & $79 \%$ \\
\hline What did you like least about online classes? & Open answer & $77 \%$ \\
\hline What would you improve? & Open answer & $62 \%$ \\
\hline
\end{tabular}

\section{Results and Discussions}

According to the survey, $75 \%$ of the students do not feel comfortable studying at home as this environment does not facilitate concentration for an appropriate learning. In spite of that, compared to the previous part of the Academic year (from the 4th of February until the 28th of March) during which classes were face-to-face and the attendance rate of student was of about $73 \%$, an increase in the student attendance to the virtual classes has been detected, reaching out almost the $100 \%$. On the other side, $80 \%$ of students has identified as a crucial factor for the learning process the face-to-face interaction with their classmates and the professor. The feeling of isolation is one of the aspects that has been deeply 
investigated worldwide (Casares \& Khan, 2020), (Banerjee \& Rai, 2020) being identified as one of the major obstacles to the diffusion of the e-learning.

The results show as about $67 \%$ of students feel a medium-high level of isolation attending on-line classes, as shown in Fig.3-a. The importance to have access to the recorded on-line class has been assessed as very important by $87 \%$ of students. In addition, $80 \%$ of the survey pool has considered that the team work carried out during the on-line class was very useful to improve their knowledge. $70 \%$ of participants was medium-highly satisfied by the proposed model of e-learning class (Fig. 3-b).
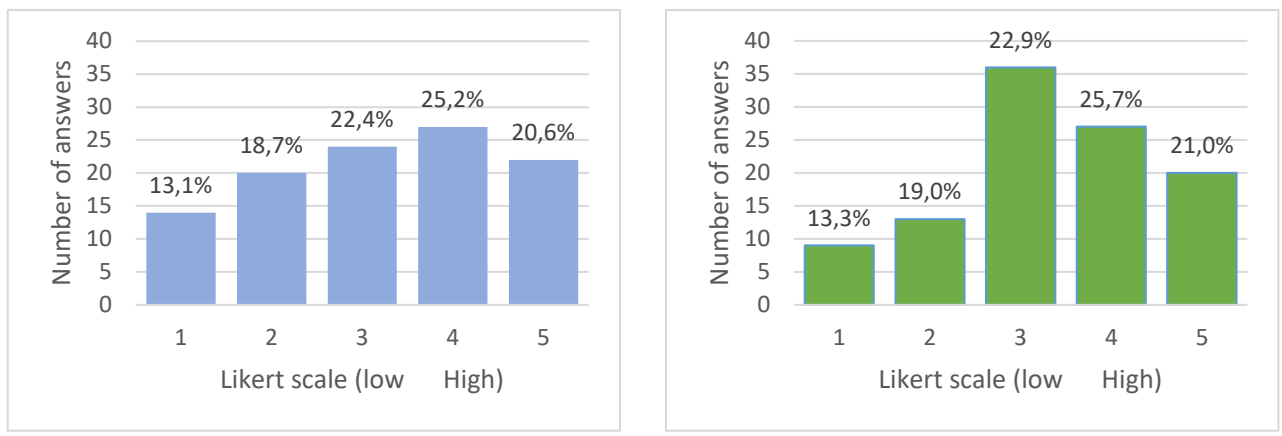

Fig. 3 Level of Student isolation (a) and student's satisfaction (b)

The electronic black-board, the video of the class and Kahoot have been considered the most useful tools to stimulate learning (Fig.4).

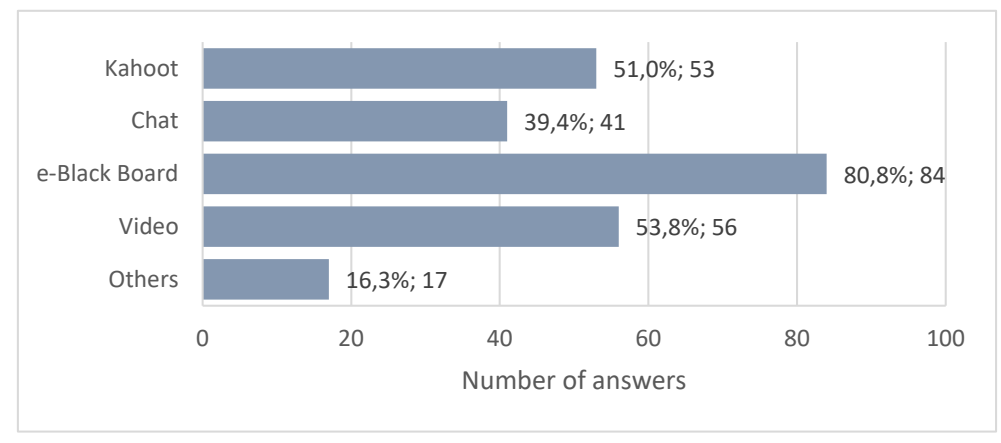

Fig. 4 Tools for the e-learning

The major benefits and limitations identified by the students while participating into the e-learning classes have been summarized as follows:

\section{Advantages}

- Recording of the class with the possibility to watch later and review topics at any desired time $(95 \%)$

- $\quad$ Flexibility of e-learning: You can study anywhere and at any time (80\%) 
- High teachers' commitment and ability to adapt to this emergency situation (15\%)

- The comfort of not having to travel to the University $(5 \%)$

- To have a wide spectrum of education materials with a better quality (5\%)

- The use of dynamic tools like Kahoot that generate interest and have a better impact on students ${ }^{\text {ee }}$ learning. (5\%)

- $\quad$ Teacher tutoring is more fluent and useful $(2 \%)$

\section{Disadvantages}

- Low interaction with professors $(20 \%)$

- Low interaction between students $(20 \%)$

- $\quad$ Students ${ }^{\text {ee }}$ isolation (19\%)

- Low participation of students (19\%)

- Poor internet connection during the class and loss of the visual image (16\%)

- Strong difficult to concentrate of students during the virtual class (15\%)

- The quality of the explanation is reduced due to the difficulty of e-blackboard on the screen, poor video or audio, etc. $(12 \%)$

- Excessive load of work, face-to-face activities have been replaced by a lot of new assignments $(10 \%)$

- Low empathy of professors about the situation generated by COVID-19 and the psychologic effects on student's life. Professors should have reduced the level of difficulty to empathize with the students $(8 \%)$

- The exams and online evaluations are much more stressful (7\%)

- $\quad$ Lack of immediate feedback during virtual classes. In face-to-face class, you can ask it easily and quickly in a moment at the end of the class. On-line communication is much more complicated (4\%)

Finally, students ${ }^{\text {ee }}$ suggestions in terms of recommendations and improvements for the future on-line classes since the student perspective are enclosed below:

\section{Recommendations and Improvements suggested by students:}

- Increase students"e participation through collaborative work (e.g. Kahoot) $(30 \%)$

- Change the traditional format of the exams by an academic work and increase the time for on-line test completion (24\%)

- Class videos should be available from the first beginning and the material should be chronologically organized (22\%)

- Improve the internet connection to prevent failures during on-line sessions and provide professors with IT tools of higher quality (electronic blackboard, etc.) $(20 \%)$

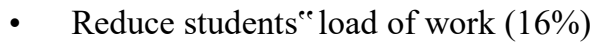

- Increase professors ${ }^{e e}$ empathy and commitment (5\%) 


\section{Conclusions}

The coming of COVID-19 has produced a deep impact in the way in which University education was developed. Students and professors have been forced to adapt themselves in a very short period of time to this situation, which has motivated the appearing of new educational methodologies based on remote education. This article presents the experience of the authors related to this issue in master degree classes at the Polytechnic University of Valencia. This methodology made possible the fast adaptation to the new situation, which has been facilitated by the high level of IT services and tools already available at UPV. The methodology has been based on the performance of on-line classes which were recorded to allow the later visualization of students; the implementation of remote team activities such as problem solving and competitions thanks to such tools as Kahoot; and the on-line evaluation by means of tests and practical exercises by means of the ERP system "Poliformat". This research concludes with a survey in which students have evidenced the advantages and disadvantages of on-line education that, in general and from a student perspective, has been successfully applied during this pandemic crisis.

\section{Acknowledgments}

This work was supported in part by the regional public administration of Valencia under the grant ACIF/2018/106.

\section{References}

Banerjee, D., \& Rai, M. (2020). Social isolation in Covid-19: The impact of loneliness. International Journal of Social Psychiatry, 1-3. doi:10.1177/0020764020922269

Boletín Oficial del Estado. (2020). Real Decreto-ley 8/2020, de 17 de marzo, de medidas urgentes extraordinarias para hacer frente al impacto económico y social del COVID-19. Jefatura del Estado. Madrid: BOE. Retrieved from https://www.boe.es/boe/dias/2020/03/18/pdfs/BOE-A2020-3824.pdf

Casares, M., \& Khan, H. (2020). A Dynamic Model of COVID-19: Contagion and Implications of Isolation Enforcement. Pamplona: Universidad Pública de Navarra.

Fell, M. J., Pagel, L., Chen, C.-f., Goldberg, M. H., Herberz, M., Huebner, G. M., . . Hanel, U. J. (2020). Validity of energy social research during and after COVID-19: challenges, considerations, and responses. Energy Research and Social Science, In-Press. doi:https://doi.org/10.1016/j.erss.2020.101646 
Gewin, V. (2020, April 9). Five tips for moving teaching online as COVID-19 takes hold. Nature, 580, 295-296.

Nicola, M., Alsafi, Z., Sohrabi, C., Kerwan, A., Al-Jabir, A., Iosifidis, C., . . Agha, R. (2020). The socio-economic implications of the coronavirus pandemic (COVID-19): a review. International Journal of Surgery, 78, 185-193.

Soland, J., Kuhfeld, M., Tarasawa, B., Johnson, A., Ruzek, E., \& Lui, J. (2020, May 27). The impact of COVID-19 on student achievement and what it may mean for educators. Brown Center Chalkboard. Retrieved June 14, 2020 